\title{
GLOBAL DIMENSION OF VALUATION RINGS
}

\author{
B. L. OSOFSKY( $\left.{ }^{1}\right)$
}

1. Introduction. Let $R$ be a ring with identity 1 . Let $\mathfrak{M}_{R}$ denote the category of unital right $R$-modules. All modules will be in $\mathfrak{M}_{R}$.

Let $M \in \mathfrak{M}_{R}$. A projective (injective) resolution of $M$ is an exact sequence:

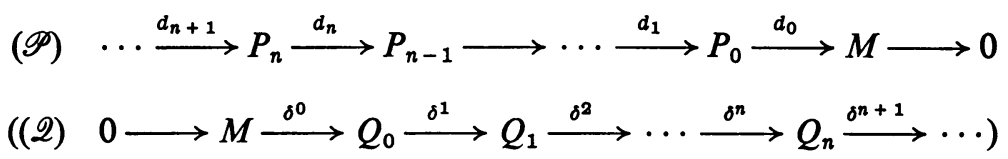

where, for all $i \geqq 0, P_{i}$ is projective in $\mathfrak{M}_{R}\left(Q_{i}\right.$ is injective in $\left.\mathfrak{M}_{R}\right) . \mathscr{P}(\mathscr{Q})$ has length $n$ if $P_{i}=0\left(Q_{i}=0\right)$ for all $i>n$, otherwise it has length $\infty$. The projective (injective) dimension of $M$, written $\operatorname{pd}(M)(\operatorname{id}(M))$ is defined by:

$$
\begin{aligned}
& \operatorname{pd}(M)=\inf \{\text { length } \mathscr{P} \mid \mathscr{P} \text { a projective resolution of } M\} ; \\
& (\operatorname{id}(M)=\inf \{\text { length } \mathscr{Q} \mid \mathscr{Q} \text { an injective resolution of } M\}) .
\end{aligned}
$$

Then

$$
\begin{aligned}
& \operatorname{pd}(M)=n \Leftrightarrow(\forall \mathscr{P})\left[\text { kernel } d_{n-1}\right. \text { is projective], } \\
& \left(\operatorname{id}(M)=n \Leftrightarrow(\forall \mathscr{Q})\left[\text { image } \delta^{n}\right. \text { is injective]). }\right.
\end{aligned}
$$

The right global dimension of $R, \operatorname{gl} . \mathrm{d}(R)$, is given by

$$
\operatorname{gl.d}(R)=\sup \left\{\operatorname{pd}(M) \mid M \in \mathfrak{M}_{R}\right\} .
$$

The Global Dimension Theorem (Auslander [1]) enables one to look only at dimensions of right ideals of $R$ or of cyclic right $R$-modules, for

$$
\begin{aligned}
\operatorname{gl.d}(R) & =\sup \{\operatorname{pd}(R / I) \mid I \text { a right ideal of } R\} \\
& =0 \text { or } 1+\sup \{\operatorname{pd}(I) \mid I \text { a right ideal of } R\} .
\end{aligned}
$$

This global dimension, defined in terms of projective resolutions of modules, turns out to be definable also in terms of injective resolutions, for we have

$$
\operatorname{gl.d}(R)=\sup \left\{\operatorname{id}(M) \mid M \in \mathfrak{M}_{R}\right\} .
$$

(See $[4$, p. 111].) In general, there is no analogy to the Global Dimension Theorem in terms of injective dimensions of cyclic modules or right ideals, although if $R$ is

Presented to the Society, August 23, 1965; received by the editors August 25, 1965.

( $\left.{ }^{1}\right)$ The author gratefully acknowledges support from the National Science Foundation under grant GP-4226. 
right perfect we do get one (\$3). If $R$ is right noetherian, then the global dimension of $R$ is the supremum of the injective dimensions of cyclic modules. Moreover, if every cyclic $R$-module has injective dimension $=0$, then $R$ is semisimple artinian, so $\operatorname{gl} . \mathrm{d}(R)=0$ (see Osofsky [13]).

However, if

$$
\sup \{\operatorname{id}(R / I) \mid I \text { a right ideal of } R\} \leqq 1
$$

nothing whatsoever can be said about the global dimension of $R$. For, by a theorem of Matlis [10], this property is true for maximally complete valuation rings. In $\$ 2$ we show that the global dimension of a valuation ring may be any nonnegative integer or $\infty$, depending only on the order type of the set of principal ideals of $R$ (hence the value group).

2. Global dimension of valuation rings. In this section, $R$ will denote a valuation ring. We take as our definition:

$R$ is a valuation ring if and only if $R$ has no zero divisors, and the lattice of right ideals of $R$ is linearly ordered.

Remarks. (See Schilling [15], Chapter 1 as a general reference on valuation rings.)

(i) Technically, we have defined "right valuation rings." $R$ is a valuation ring if and only if $R$ is a domain and both the lattice of right ideals and the lattice of left ideals are linearly ordered. (Many authors also require $R$ to be commutative.) A twisted power series ring is a right valuation ring but not a valuation ring if the twisting isomorphism is not onto. However, for convenience, we will drop the term "right" from the phrases "right valuation ring" and "right ideal," even though our theorems apply to a wider class of rings than valuation rings in the accepted sense.

(ii) Every finitely generated ideal of $R$ is principal, and hence a free $R$-module.

(iii) If $x, y \in R$, then either $x^{-1} y$ or $y^{-1} x \in R$, where inverses are taken in the field of quotients of the Ore domain $R$ (see Ore [12]).

(iv) The set of nonunits of $R$ form an ideal which will be denoted by $J$ (for Jacobson radical).

A basic tool in calculating the global dimension of $R$ is:

TheOREM (KAPLANSKY [8]). Let $P$ be a projective module over a ring whose nonunits form an ideal. Then $P$ is free.

COROLlaRY. Every projective right $R$-module is free.

Proof. Combine Kaplansky's theorem with remark (iv).

The remainder of our preliminary development will concern ordered sets, for it is the linear ordering of the ideals of $R$ which tremendously simplifies the calculation of its global dimension.

Definition. If $S$ and $T$ are linearly ordered sets, we say that $S$ is cofinal in $T$ if 
there is an order-preserving one-to-one function $\phi: S \rightarrow T$ such that for all $t \in T$, there is an $s \in S$ with $t \leqq \phi(s)$. If $S \subseteq T$, we will assume $\phi$ is the inclusion map. We say that $\phi$ embeds $S$ cofinally in $T$.

We set the ordinal $0=\varnothing$, and identify each ordinal with the set of those ordinals less than it.

DEFINITION. An ordinal $\alpha$ is called regular if it is infinite and no ordinal less than $\alpha$ is cofinal in $\alpha$.

Notation. For each ordinal $\alpha$, let $\Omega_{\alpha}$ denote the first ordinal of cardinality $\boldsymbol{\aleph}_{\alpha}$. For example, $\Omega_{0}=\omega, \Omega_{1}=$ the first uncountable ordinal. Set $\Omega_{-1}=1$, where -1 is considered less than any ordinal.

For any set $A$, let $|A|$ denote the cardinality of $A$.

LEMMA 1. Let $S$ be a linearly ordered set, and let $\alpha$ be the first ordinal of cardinality $|S|$. Then there exists an ordinal $\leqq \alpha$ which is cofinal in $S$.

Proof. Let $\psi: \alpha \rightarrow S$ be a one-to-one correspondence (ignoring order). Set $\phi(0)=\psi(0)$. Assume the order-preserving, one-to-one function $\phi$ has been defined for all $\beta<\gamma \in \alpha$. If, for all $s \in S$, there is a $\beta<\gamma$ such that $\phi(\beta) \geqq s$, then $\phi$ embeds $\gamma$ cofinally in $S$. If not, define

$$
\phi(\gamma)=\psi(\inf \{\delta<\alpha \mid \psi(\delta)>\phi(\beta) \text { for all } \beta<\gamma\}) .
$$

Clearly $\phi$ is order-preserving and one-to-one on the ordinal $\gamma+1$.

If this process does not terminate because some ordinal $\gamma<\alpha$ has been embedded cofinally in $S$, then $\psi^{-1} \phi\{\beta<\alpha\}=A$ is a subset of $\alpha$ with $|A|=|\alpha|$. Since every ordinal $<\alpha$ has smaller cardinality than this, the supremum of $A$ must be $\alpha$ (or $\alpha-1$ ). Let $s=\psi(\beta) \in S$, and let $\gamma \in A$ be such that $\beta \leqq \gamma<\alpha$. Then $\psi(\gamma)=\phi(\delta)$ for some $\delta<\alpha$. Since $\beta \leqq \gamma, \psi(\beta) \leqq \psi(\gamma)$ by definition of $\phi(\delta)$; i.e., $s \leqq \phi(\delta)$. Thus $\phi$ embeds $\alpha$ cofinally in $S$.

LEMMA 2. Any regular ordinal is of the form $\Omega_{\alpha}$ for some $\alpha . \Omega_{\alpha}$ is a regular ordinal $\Leftrightarrow \boldsymbol{\aleph}_{\alpha}$ is strictly greater than any sum of less than $\boldsymbol{\aleph}_{\alpha}$ sets, each of cardinality $<\boldsymbol{\aleph}_{\alpha}$. If $\alpha=0$ or $\beta+1$, then $\Omega_{\alpha}$ is regular.

Proof. The first statement is an immediate consequence of Lemma 1. For the second, see Sierpinski [16, p. 405], or Tarski [17, p. 69]. The third statement follows from the second. The existence or nonexistence of regular ordinals of the form $\Omega_{\alpha}$ where $\alpha$ is a limit ordinal has not been shown.

Lemma 3. Let $S$ be a linearly ordered set such that $\Omega_{\alpha}$ is cofinal in $S$, where $\Omega_{\alpha}$ is a regular ordinal. If $T \subseteq S$ is cofinal in $S$, then $|T| \geqq \aleph_{\alpha}$.

Proof. Let $\phi$ embed $\Omega_{\alpha}$ cofinally in $S$. For each $t \in T$, let

$$
\psi(t)=\inf \left\{\beta<\Omega_{\alpha} \mid \phi(\beta)>t\right\} .
$$

$\psi$ is defined on all of $T$ since $\phi$ embeds the limit ordinal $\Omega_{\alpha}$ cofinally in $S$. Moreover, $|\psi(T)| \leqq|T|$. If $|T|<\aleph_{\alpha}, \psi(T)$ cannot be cofinal in $\Omega_{\alpha}$ by the regularity of $\Omega_{\alpha}$. 
Then $\sup (\psi(T))<\Omega_{\alpha}$. But $\phi(\sup (\psi(T))) \geqq \phi(\psi(t))>t$ for all $t \in T$. Hence $T$ is not cofinal in $S$.

For any set $A$, let $\mathfrak{F}(A)$ denote the set of all finite subsets of $A$.

LEMMA 4. Let $\alpha$ be a regular ordinal $>\Omega_{0}, \beta$ a limit ordinal $<\alpha$. Let

$$
\Lambda: \mathfrak{F}(\alpha) \rightarrow \mathfrak{F}(\alpha) \text {. }
$$

Then there exists an initial segment $S \subset \alpha$ such that

(a) $\beta$ is cofinal in $S$.

(b) $\Lambda(F) \subseteq S$ for all $F \in \mathfrak{F}(S)$.

Proof. We use transfinite induction to construct a collection of initial segments $\phi(\gamma)$ of $\alpha$ for all $\gamma<\beta$ such that

(i) $\phi(\gamma)$ properly contains $\phi(\delta)$ for all $\delta<\gamma$.

(ii) If $\gamma=\delta+1$, then $\phi(\gamma) \supset \Lambda(F)$ for all $F \in \mathfrak{F}(\phi(\delta))$, and $\phi(\gamma)$ has a largest element.

(iii) $|\phi(\gamma)|<|\alpha|$.

Set $\phi(0)=\varnothing$. Assume $\phi(\delta)$ has been defined for all $\delta<\gamma$. If $\gamma$ is a limit ordinal, set $\phi(\gamma)=\bigcup_{\delta<\gamma} \phi(\delta)$. Since $\alpha$ is regular, (iii) holds by Lemma 2. If $\delta<\gamma, \delta+1<\gamma$ so (i) holds.

If $\gamma=\delta+1,|\phi(\delta)|<|\alpha|$ implies

$$
|\mathfrak{F}(\phi(\delta))| \leqq \sum_{m=1}^{\infty}|\phi(\delta)|^{m}=\aleph_{0}|\phi(\delta)|<|\alpha| .
$$

Then $\left|\bigcup_{F \in \mathfrak{F}(\phi(\delta))} \Lambda(F)\right| \leqq \aleph_{0}|\mathfrak{F}(\phi(\delta))|<|\alpha|$, so

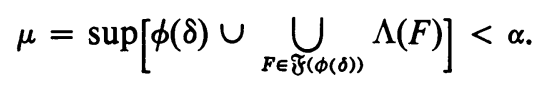

Set $\phi(\gamma)=\{\nu \leqq \mu+1\}$. Then (i), (ii), and (iii) clearly hold.

Set

$$
S=\bigcup_{\gamma<\beta} \phi(\gamma)
$$

$S$ is an initial segment of $\alpha$ since it is a union of initial segments. The map $\gamma \rightarrow \sup [\phi(\gamma+1)]$ embeds $\beta$ cofinally in $S$ so (a) holds. If $F \in \mathfrak{F}(S)$, there is a $\gamma<\beta$ such that $F \subseteq \phi(\gamma)$. Then $\Lambda(F) \subseteq \phi(\gamma+1) \subset S$ so (b) holds.

We now return to our valuation ring $R$. Let $I$ be an ideal of $R$, and set

$$
S=\{x R \mid x \in I\} .
$$

$S$ is linearly ordered by inclusion. By Lemma 1, there exists an ordinal $\alpha$ and an order preserving map $\beta \rightarrow x_{\beta}$ such that $T=\left\{x_{\beta} R \mid \beta<\alpha\right\} \subseteq S$ is cofinal in $S$.

Let $x \in I$. Then there exists a $\beta<\alpha$ such that $x_{\beta} R \supseteq x R$, so $I \subseteq \sum_{\beta<\alpha} x_{\beta} R$. Since $x_{\beta} R \subseteq I$, the reverse inclusion holds and

$$
I=\sum_{\beta<\alpha} x_{\beta} R
$$


Also, for $\beta<\gamma, x_{\beta} R$ is properly contained in $x_{y} R$. Hence $x_{\gamma}^{-1} x_{\beta} \in J$, the ideal of nonunits.

Let $P_{n}$ be the free $R$-module generated by symbols

$$
\left(\beta_{0}, \beta_{1}, \ldots, \beta_{n}\right) \quad \beta_{0}<\beta_{1}<\cdots<\beta_{n}<\alpha .
$$

That is, $P_{n}=\sum \oplus\left(\beta_{0}, \beta_{1}, \ldots, \beta_{n}\right) R$. Set $P_{-1}=I$.

Define $d_{n}: P_{n} \rightarrow P_{n-1}$ by

$$
\begin{aligned}
d_{0}\left(\beta_{0}\right) & =x_{\beta_{0}} \\
d_{n}\left(\beta_{0}, \ldots, \beta_{n}\right) & =\sum_{i=0}^{n-1}(-1)^{i}\left(\beta_{0}, \ldots, \hat{\beta}_{n-i}, \ldots, \beta_{n}\right)+(-1)^{n}\left(\beta_{1}, \ldots, \beta_{n}\right) x_{\beta_{1}}^{-1} x_{\beta_{0}}
\end{aligned}
$$

where $\hat{\beta}_{n-i}$ means omit $\beta_{n-i}$.

Our definitions of $P_{n}$ and $d_{n}$ look much like the standard combinatorial topology definitions of an abstract complex and boundary operator. Indeed, topological techniques carry over exactly to show:

LEMMA 5.

$$
\text { (P) } \quad \cdots \stackrel{d_{n+1}}{\longrightarrow} P_{n} \stackrel{d_{n}}{\longrightarrow} P_{n-1} \longrightarrow \cdots \stackrel{d_{1}}{\longrightarrow} P_{0} \stackrel{d_{0}}{\longrightarrow} I \longrightarrow 0
$$

is a projective resolution of $I$.

Proof. (i) $\mathscr{P}$ is a complex.

$$
d_{0} d_{1}\left(\beta_{0}, \beta_{1}\right)=d_{0}\left(\beta_{0}-\beta_{1} x_{\beta_{1}}^{-1} x_{\beta_{0}}\right)=x_{\beta_{0}}-x_{\beta_{1}} x_{\beta_{1}}^{-1} x_{\beta_{0}}=0 .
$$

For $n>1$,

$$
\begin{aligned}
d_{n-1} d_{n}\left(\beta_{0}, \ldots, \beta_{n}\right)= & d_{n-1}\left[\sum_{i=0}^{n-1}(-1)^{i}\left(\beta_{0}, \ldots, \hat{\beta}_{n-i}, \ldots, \beta_{n}\right)\right. \\
& \left.+(-1)^{n}\left(\beta_{1}, \ldots, \beta_{n}\right) x_{\beta_{1}}^{-1} x_{\beta_{0}}\right] \\
=\sum_{i=0}^{n-1}(-1)^{i}\left[\sum_{j=0}^{i-1}(-1)^{j}\left(\beta_{0}, \ldots, \hat{\beta}_{n-i}, \ldots, \hat{\beta}_{n-j}, \ldots, \beta_{n}\right)\right. & \\
& \left.+\sum_{j=i+1}^{n-1}(-1)^{j-1}\left(\beta_{0}, \ldots, \hat{\beta}_{n-j}, \ldots, \hat{\beta}_{n-i}, \ldots, \beta_{n}\right)\right] \\
& +(-1)^{n-1} \sum_{i=0}^{n-2}(-1)^{i}\left(\beta_{1}, \ldots, \hat{\beta}_{n-i}, \ldots, \beta_{n}\right) x_{\beta_{1}}^{-1} x_{\beta_{0}} \\
& +(-1)^{n-1}(-1)^{n-1}\left(\beta_{2}, \ldots, \beta_{n}\right) x_{\beta_{2}}^{-1} x_{\beta_{0}} \\
& +(-1)^{n} \sum_{i=0}^{n-2}(-1)^{i}\left(\beta_{1}, \ldots, \hat{\beta}_{n-i}, \ldots, \beta_{n}\right) x_{\beta_{1}}^{-1} x_{\beta_{0}} \\
& +(-1)^{n}(-1)^{n-1}\left(\beta_{2}, \ldots, \beta_{n}\right) x_{\beta_{2}}^{-1} x_{\beta_{1}} x_{\beta_{1}}^{-1} x_{\beta_{0}} .
\end{aligned}
$$


We observe that every term occurs twice with coefficients differing only by sign. Hence $d_{n-1} d_{n}=0$.

(ii) $\mathscr{P}$ is exact.

$\mathscr{P}$ is exact at $I$ since $I=\sum_{\beta<\alpha} x_{\beta} R$.

Let $\sum_{i=1}^{m}\left(\beta_{i, 0}, \ldots, \beta_{i, n}\right) r_{i} \in P_{n}$ for $n \geqq 0$, and let $\beta$ be greater than $\beta_{i, n}$ for all $i$. If $n=-1$, let $r \in x_{\beta} R$. Define

$$
\begin{aligned}
{\left[\sum_{i=1}^{m}\left(\beta_{i, 0}, \ldots, \beta_{i, n}\right) r_{i}\right] * \beta } & =\sum_{i=1}^{m}\left(\beta_{i, 0}, \ldots, \beta_{i, n}, \beta\right) r_{i}, \\
r * \beta & =(\beta) x_{\beta}^{-1} r .
\end{aligned}
$$

Then $* \beta$ is a monomorphism from $\sum_{\beta_{n}<\beta}\left(\beta_{0}, \ldots, \beta_{n}\right) R \rightarrow P_{n+1}\left(\right.$ or $\left.x_{\beta} R \rightarrow P_{0}\right)$. We observe that

$$
d_{n+1}\left[\left(\beta_{0}, \ldots, \beta_{n}\right) * \beta\right]=\left(\beta_{0}, \ldots, \beta_{n}\right)-\left[d_{n}\left(\beta_{0}, \ldots, \beta_{n}\right)\right] * \beta
$$

for all $n \geqq 0$.

Now let $p=\sum_{i=1}^{m}\left(\beta_{i, 0}, \ldots, \beta_{i, n}\right) r_{i} \in \operatorname{kernel} d_{n}$, and let $\beta=\max \left\{\beta_{i, n} \mid 1 \leqq i \leqq m\right\}$. Then $p=p^{\prime}+p^{\prime \prime} * \beta$ where $p^{\prime} \in \sum_{\beta_{n}<\beta}\left(\beta_{0}, \ldots, \beta_{n}\right) R$ and $p^{\prime \prime} \in P_{n-1}$. Then

$$
\begin{aligned}
p-d_{n+1}\left(p^{\prime} * \beta\right) & =p^{\prime}+p^{\prime \prime} * \beta-\left[p^{\prime}-\left(d_{n}\left(p^{\prime}\right)\right) * \beta\right] \\
& =\left(p^{\prime \prime}+d_{n}\left(p^{\prime}\right)\right) * \beta .
\end{aligned}
$$

Since $\mathscr{P}$ is a complex, $d_{n}\left(p-d_{n+1}\left(p^{\prime} * \beta\right)\right)=0$ so

$$
\begin{aligned}
& \left(p^{\prime \prime}+d_{n}\left(p^{\prime}\right)\right)-\left[d_{n-1}\left(p^{\prime \prime}+d_{n}\left(p^{\prime}\right)\right)\right] * \beta=0 \text { if } n>0 \text {, } \\
& x_{\beta} x_{\beta}^{-1}\left(p^{\prime \prime}+d_{n}\left(p^{\prime}\right)\right)=0 \text { if } n=0 \text {. }
\end{aligned}
$$

If $n=0, p^{\prime \prime}+d_{n}\left(p^{\prime}\right)=0$ since $x_{\beta} x_{\beta}^{-1}=1$.

If $n>0$, distinct $n$-tuples in $P_{n-1}$ are independent, and no $n$-tuple in $p^{\prime \prime}+d_{n}\left(p^{\prime}\right)$ involves $\beta$, so $p^{\prime \prime}+d_{n}\left(p^{\prime}\right)=0$. Hence $p=d_{n+1}\left(p^{\prime} * \beta\right)$. Note that it was not necessary to involve new ordinals in the element whose boundary is $p$; all occur in $p$.

We now have the projective resolution $\mathscr{P}$ of $I$ which we can use to calculate the projective dimension of $I$.

We note:

(i) $\operatorname{pd}(I) \leqq n+1 \Leftrightarrow$ kernel $d_{m}=$ image $d_{m+1}$ is projective for all $m \geqq n$. (See [4, p. 110].)

(ii) If $\gamma$ is cofinal in $\alpha$, then $\gamma$ is cofinal in $S$ and we may use it, rather than $\alpha$, to calculate $\operatorname{pd}(I)$.

(iii) Let $\gamma<\Omega_{n}$ for $n \in \omega$. If $n>0$, by Lemma 1, some ordinal $\leqq \Omega_{n-1}$ is cofinal in $\gamma$. If $n=0, \Omega_{-1}$ is cofinal in $\gamma$.

(iv) $\Omega_{n}$ is cofinal in $S$ if and only if $I$ has a generating set consisting of $\boldsymbol{K}_{n}$ elements, and no set of fewer elements generates $I$.

LEMMA 6. $\Omega_{-1}$ is cofinal in $S \Leftrightarrow \operatorname{pd}(I)=0$. 
Proof. $\operatorname{pd}(I)=0 \Leftrightarrow I$ is projective $\Leftrightarrow I$ has a free basis containing an element $y$ $\Leftrightarrow$ for some $y$, no $x_{\beta} R$ properly contains $y R \Leftrightarrow I$ is principal $\Leftrightarrow 1=\Omega_{-1}$ is cofinal in $S$.

LEMMA 7. Let $n \geqq 0$. Assume for all $\gamma<\alpha, d_{n}\left(\sum_{\beta_{n}<\gamma}\left(\beta_{0}, \ldots, \beta_{n}\right) R\right)$ is projective. Then $d_{n+1}\left(P_{n+1}\right)$ is projective.

Proof. We define, for each $\gamma<\alpha$, a set $T_{\gamma}$ such that:

(a) For $\beta<\gamma, T_{\beta} \subset T_{\gamma}$.

(b) $T_{y}$ is a free basis for $d_{n+1}\left[\sum_{\beta_{n+1} \leqq \gamma}\left(\beta_{0}, \ldots, \beta_{n+1}\right) R\right]$.

Set $T_{0}=\varnothing$. Assume $T_{\beta}$ has been defined for all $\beta<\gamma$. By hypothesis, $d_{n}\left[\sum_{\beta_{n}<\gamma}\left(\beta_{0}, \ldots, \beta_{n}\right) R\right]$ is projective. By the corollary to Kaplansky's theorem, it has a free basis, say $\left\{d_{n} p_{i} \mid p_{i} \in \sum_{\beta_{n}<\gamma}\left(\beta_{0}, \ldots, \beta_{n}\right) R\right\}$. Set

Clearly (a) holds.

$$
T_{\gamma}=\bigcup_{\beta<\gamma} T_{\beta} \cup\left\{d_{n+1}\left(p_{i} * \gamma\right)\right\}
$$

Let $\sum_{j=1}^{m} t_{j} s_{j}+\sum_{h=1}^{k} d_{n+1}\left(p_{i_{h}} * \gamma\right) r_{h}=0$, where $\left\{t_{j}\right\} \subseteq \bigcup_{\beta<\gamma} T_{\beta}$. Then

$$
\sum_{h=1}^{k}\left(d_{n}\left(p_{i_{h}}\right) * \gamma\right) r_{h}=0
$$

since these are the only $(n+1)$-tuples involving $\gamma$. Thus $\sum_{h=1}^{k}\left(d_{n}\left(p_{i_{h}}\right)\right) r_{h}=0$, and by the independence of $\left\{d_{n}\left(p_{i}\right)\right\}, r_{h}=0$ for all $h, 1 \leqq h \leqq k$. Since $\left\{t_{j} \mid 1 \leqq j \leqq m\right\}$ is finite, there is a $\beta<\gamma$ such that $\left\{t_{j}\right\} \subseteq T_{\beta}$. Since $T_{\beta}$ consists of independent elements, $s_{j}=0$ for all $1 \leqq j \leqq m$. Hence $T_{\gamma}$ consists of independent elements.

Let $p \in d_{n+1}\left[\sum_{\beta_{n+1} \leqq \gamma}\left(\beta_{0}, \ldots, \beta_{n+1}\right) R\right]$. Then $p=d_{n+1}(q * \gamma)$ for some

$$
q \in \sum_{\beta_{n}<\gamma}\left(\beta_{0}, \ldots, \beta_{n}\right) R \text {. }
$$

Since $\left\{d_{n}\left(p_{i}\right)\right\}$ spans $d_{n}\left[\sum_{\beta_{n}<y}\left(\beta_{0}, \ldots, \beta_{n}\right) R\right], d_{n}(q)=\sum_{h=1}^{k} d_{n}\left(p_{i_{n}}\right) r_{h}$. Then

$$
\begin{aligned}
t= & p-\sum_{n=1}^{k}\left[d_{n+1}\left(p_{i_{h}} * \gamma\right)\right] r_{h}=q-\left[d_{n}(q)\right] * \gamma \\
& -\sum_{h=1}^{k} p_{i_{h}} r_{h}+\sum_{h=1}^{k}\left[d_{n}\left(p_{i_{h}}\right) r_{h}\right] * \gamma=q-\sum_{h=1}^{k} p_{i_{h}} r_{h}
\end{aligned}
$$

is in $d_{n+1}\left[\sum_{\beta_{n+1} \leqq \beta}\left(\beta_{0}, \ldots, \beta_{n+1}\right) R\right]$ for some $\beta<\gamma$. Then $t$ is a sum of elements in $T_{\beta}$, and $T_{y}$ spans $d_{n+1}\left(\sum_{\beta_{n+1} \leqq y}\left(\beta_{0}, \ldots, \beta_{n+1}\right) R\right)$. Thus (b) holds.

Set

$$
T=\bigcup_{\gamma<\alpha} T_{\gamma}
$$

Since any finite set of elements in $d_{n+1}\left(P_{n+1}\right)$ is in $d_{n+1}\left(\sum_{\beta_{n+1} \leq \gamma}\left(\beta_{0}, \ldots, \beta_{n}\right) R\right)$ for some $\gamma<\alpha$, and $T_{\gamma}$ is a free basis for this set, $T$ must be a free basis for $d_{n+1}\left(P_{n+1}\right)$, which is therefore projective.

LEMMA 8. Let $\gamma<\alpha, n \geqq 1$. Then $d_{n}\left[\sum_{\beta_{n} \leqq \gamma}\left(\beta_{0}, \ldots, \beta_{n}\right) R\right]$ is a direct summand of $d_{n}\left(P_{n}\right)$. 
Proof. Let $p \in d_{n}\left(P_{n}\right)$. Then

$$
p=d_{n}\left(p_{1} * \gamma\right)+d_{n}\left(p_{2} * \beta\right)
$$

where $p_{1}$ is the sum of those terms in $p$ with last coordinate $<\gamma$, and each $n-1$ tuple in $p_{2}$ ends with an ordinal $\geqq \gamma$. Consider the map

$$
\pi: p \rightarrow d_{n}\left(p_{1} * \gamma\right) \text {. }
$$

$\pi$ is a composition of three $R$-homomorphisms-a projection in $P_{n-1}$ followed by $* \gamma$ followed by $d_{n}$. Thus $\pi$ is an $R$-homomorphism. Moreover, $\pi^{2}=\pi$, so $\pi$ is a projection onto

$$
\text { range } \pi=d_{n}\left(\sum_{\beta_{n} \leqq \gamma}\left(\beta_{0}, \ldots, \beta_{n}\right) R\right)
$$

Then

$$
d_{n}\left(P_{n}\right)=d_{n}\left(\sum_{\beta_{n} \leqq \gamma}\left(\beta_{0}, \ldots, \beta_{n}\right) R\right) \oplus \text { kernel } \pi .
$$

LEMMA 9. Let $n \geqq 1$, and assume $d_{n}\left(P_{n}\right)$ is projective. If, for $\gamma<\alpha$,

$$
d_{n}\left(\sum_{\beta_{n}<\gamma}\left(\beta_{0}, \ldots, \beta_{n}\right) R\right)
$$

is a direct summand of $d_{n}\left(P_{n}\right)$, then $d_{n-1}\left(\sum_{\beta_{n-1}<\gamma}\left(\beta_{0}, \ldots, \beta_{n-1}\right) R\right)$ is projective.

Proof. Let

$$
d_{n}\left(P_{n}\right)=d_{n}\left(\sum_{\beta_{n}<\gamma}\left(\beta_{0}, \ldots, \beta_{n}\right) R\right) \oplus M,
$$

and let $\pi$ be the projection of Lemma 8 . Then

$$
\text { range } \pi=d_{n}\left(\sum_{\beta_{n}<\gamma}\left(\beta_{0}, \ldots, \beta_{n}\right) R\right) \oplus M \cap \text { range } \pi
$$

and $M \cap$ range $\pi$ is a direct summand of a projective module; hence projective. By the corollary to Kaplansky's theorem, it has a free basis. Such a basis must be of the form $\left\{d_{n}\left(p_{i} * \gamma\right) \mid p_{i} \in \sum_{\beta_{n-1}<\gamma}\left(\beta_{0}, \ldots, \beta_{n-1}\right) R\right\}$. We claim $\left\{d_{n-1}\left(p_{i}\right)\right\}$ is a free basis for $d_{n-1}\left(\sum_{\beta_{n-1}<\gamma}\left(\beta_{0}, \ldots, \beta_{n-1}\right) R\right)$.

Let $\sum_{h=1}^{k} d_{n-1}\left(p_{i_{n}}\right) r_{h}=0$. Then

$$
\sum_{h=1}^{k} d_{n}\left(p_{i_{h}} * \gamma\right) r_{h}=\sum_{h=1}^{k} p_{i_{h}} r_{h}-\left[\sum_{n=1}^{k} d_{n-1}\left(p_{i_{h}}\right) r_{h}\right] * \gamma \in \sum_{\beta_{n}<\gamma}\left(B_{0}, \ldots, \beta_{n}\right) R \cap M=0 .
$$

By the independence of $\left\{d_{n}\left(p_{i} * \gamma\right)\right\}, r_{n}=0$ for all $h, 1 \leqq h \leqq k$. Thus $\left\{d_{n-1}\left(p_{i}\right)\right\}$ is independent.

Let $q \in \sum_{\beta_{n-1}<\gamma}\left(\beta_{0}, \ldots, \beta_{n-1}\right) R$. Then

$$
d_{n}(q * \gamma) \in \text { image } \pi=d_{n}\left(\sum_{\beta_{n}<\gamma}\left(\beta_{0}, \ldots, \beta_{n}\right) R\right)+\text { image } \pi \cap M .
$$


Hence $d_{n}(q * \gamma)=q_{1}+d_{n}\left(q_{2} * \gamma\right)$ where $q_{1} \in d_{n}\left(\sum_{\beta_{n}<\gamma}\left(\beta_{0}, \ldots, \beta_{n}\right) R\right)$ and $d_{n}\left(q_{2} * \gamma\right) \in M$. $d_{n}(q * \gamma)=q-\left(d_{n-1}(q)\right) * \gamma=q_{1}+q_{2}-\left(d_{n-1}\left(q_{2}\right)\right) * \gamma$ so $d_{n-1}(q)=d_{n-1}\left(q_{2}\right)$.

Since $\left\{d_{n}\left(p_{i} * \gamma\right)\right\}$ spans image $\pi \cap M$,

$$
\begin{aligned}
q_{2}-\left[d_{n-1}\left(q_{2}\right)\right] * \gamma & =d_{n}\left(q_{2} * \gamma\right)=\sum_{n=1}^{k} d_{n}\left(p_{i_{h}} * \gamma\right) r_{h} \\
& =\sum_{n=1}^{k} p_{i_{h}} r_{h}-\left[\sum_{h=1}^{k} d_{n-1}\left(p_{i_{h}}\right) r_{h}\right] * \gamma,
\end{aligned}
$$

so $d_{n-1}(q)=d_{n-1}\left(q_{2}\right)=\sum_{n=1}^{k} d_{n-1}\left(p_{i_{n}}\right) r_{h}$, and $\left\{d_{n-1}\left(p_{i}\right)\right\}$ spans

$$
d_{n-1}\left[\sum_{\beta_{n}-1<\gamma}\left(\beta_{0}, \ldots, \beta_{n-1}\right) R\right] \text {. }
$$

THEOREM A. For $n \geqq-1, \operatorname{pd}(I)=n+1$ if and only if $\Omega_{n}$ is cofinal in

$$
S=\{x R \mid x \in I\} .
$$

Proof. We use induction on $n$. The basis of this induction is Lemma 6. Now assume the theorem is true for all $k \leqq n$.

If $\Omega_{n+1}$ is cofinal in $S$, we may take $\alpha=\Omega_{n+1}$ in the construction of $\mathscr{P}$. Then for all $\gamma<\alpha, \operatorname{pd}\left(\sum_{\beta<\gamma} x_{\beta} R\right) \leqq n+1$ by the induction hypothesis, since Lemma 1 implies that some ordinal of the form $\Omega_{k}$ for $k \leqq n$ is cofinal in $\gamma$. Then $d_{n+1}\left[\sum_{\beta_{n+1}<\gamma}\left(\beta_{0}, \ldots, \beta_{n+1}\right) R\right]$ is projective by remark (i) preceding Lemma 6 . Hence $d_{n+2}\left(P_{n+2}\right)$ is projective by Lemma 7, so $\operatorname{pd}(I) \leqq n+2$. By Lemma $2, \Omega_{n+1}$ is regular so, for $k \leqq n, \Omega_{k}$ cannot be cofinal in $S$ by Lemma 3. By the induction hypothesis, $\operatorname{pd}(I)>n+1$. Hence $\operatorname{pd}(I)=n+2$.

Now let $\operatorname{pd}(I)=n+2$, and let $\alpha$ be the smallest ordinal cofinal in $S$. Then $\alpha$ is regular. By the induction hypothesis and Lemma $2, \alpha>\Omega_{n}$. Assume $\alpha>\Omega_{n+1}$. Let $\left\{y_{i} \mid i \in \mathscr{I}\right\}$ be a free basis for image $d_{n+2}$. Such a basis exists by the corollary to Kaplansky's theorem. Let $F \in \mathfrak{F}(\alpha)$. For $\beta_{0}<\beta_{1}<\cdots<\beta_{n+2}$, $\left\{\beta_{j}\right\} \subseteq F$, let $d_{n+2}\left(\beta_{0}, \ldots, \beta_{n+2}\right)=\sum_{i=1}^{m} y_{i} r_{i}$. Define $\Lambda\left(\left\{\beta_{j} \mid 0 \leqq j \leqq n+2\right\}\right)=\left\{\delta<\alpha \mid\right.$ for some $i, y_{i}$ has nonzero projection on an $n+2$ tuple in which the ordinal $\delta$ appears\},

$$
\Lambda(F)=\bigcup_{\{\beta, \mid 0 \leqq j \leqq n+2\} \subseteq F} \Lambda\left(\left\{\beta_{j}\right\}\right) .
$$

Then $\Lambda(F)$ is finite.

By Lemma 4, there is an ordinal $\gamma<\alpha$ such that $\Omega_{n+1}$ is cofinal in $\gamma$ and $\Lambda: \mathfrak{F}(\gamma) \rightarrow \mathfrak{F}(\gamma)$. Then

$$
d_{n+2}\left(P_{n+2}\right)=d_{n+2}\left(\sum_{\beta_{n+2}<\nu}\left(\beta_{0}, \ldots, \beta_{n+2}\right) R\right) \oplus \sum y_{i} R,
$$

where the latter sum is taken over all $y_{i}$ involving a coordinate $\geqq \gamma$. By Lemma 9 , $d_{n+1}\left(\sum_{\beta_{n+1}<\gamma}\left(\beta_{0}, \ldots, \beta_{n+1}\right) R\right)$ is projective, so $\operatorname{pd}\left(\sum_{\delta<\gamma} \gamma_{\delta} R\right) \leqq n+1$. By the induction hypothesis, there is a $k \leqq n$ such that $\Omega_{k}$ is cofinal in $\gamma$, contradicting Lemma 3. Hence $\alpha=\Omega_{n+1}$. 
Corollary 1. If $|\{x R \mid x \in R\}|=\aleph_{n}$, then $\operatorname{gl} . \mathrm{d}(R) \leqq n+2$.

Proof. Lemma 1 implies that, for any ideal $I$, there is an ordinal $\alpha=\Omega_{k}, \alpha \leqq \Omega_{n}$, such that $\alpha$ is cofinal in the set of principal subideals of $I$. By Theorem A, $\operatorname{pd}(I)$ $\leqq n+1$. By the Global Dimension Theorem, gl.d $(R) \leqq n+2$.

We note that nothing further can be said, even in the case that $n=0$, for a discrete, rank one valuation ring has global dimension 1; a discrete, rank 2 valuation ring has global dimension 2.

COROLlARY 2. Let $n$ be any nonnegative integer or $\infty$. Then there exists a valuation ring with global dimension $n$.

Proof. If $n=0$, any field will do. If $n=1$, any discrete, rank one valuation ring will do. Now assume $2 \leqq n<\infty$. Let $\Gamma$ be the additive group of all step functions from $\Omega_{n-2}$ to $Z=$ the additive group of integers; that is, $f \in \Gamma \Leftrightarrow$ there exist $0=\gamma_{0}<\gamma_{1}<\cdots<\gamma_{m}<\gamma_{m+1}=\Omega_{n-2}$ such that

$$
f(\gamma)=f\left(\gamma_{i}\right) \text { for all } \gamma \text { such that } \gamma_{i} \leqq \gamma<\gamma_{i+1} .
$$

Then $|\Gamma|=\left|\left\{\left\{\gamma_{i} \mid 1 \leqq i \leqq m<\infty\right\} \subset \Omega_{n-2}\right\}\right|,|Z|=\boldsymbol{\aleph}_{n-2} \boldsymbol{\aleph}_{0}=\boldsymbol{\aleph}_{n-2}$. Order $\Gamma$ lexicographically. For $\gamma<\Omega_{n-2}$, let $e(\gamma) \in \Gamma$ be the characteristic function of

$$
\left\{\beta \mid \gamma \leqq \beta<\Omega_{n-2}\right\} .
$$

Let $R$ be the ring of all power series in a symbol " $X$ " with exponents well ordered sequences in $\Gamma$. (See Schilling [15, p. 23].) Then $R$ is a valuation ring, and its set of principal ideals is order isomorphic to upper cuts in $\Gamma^{+}$. By Corollary 1 , gl.d $(R) \leqq n$. By Theorem A, $\operatorname{pd}\left(\sum_{y<\Omega_{n-2}} X^{e(\gamma)} R\right)=n-1$. Hence gl.d $(R) \geqq n$. We conclude gl.d $(R)=n$.

If $n=\infty$, consider the ordered group of step functions from $\Omega_{\omega+1}$ to $Z$ and proceed as above. Then, by Theorem $\mathrm{A}, \operatorname{pd}\left(\sum_{\gamma<\Omega_{\omega+1}} X^{(\gamma)} R\right)=\infty$ since $\Omega_{n}$ is not cofinal in $\Omega_{\omega+1}$ for any $n<\omega$. Thus gl.d $(R)=\infty$.

LeMMA 10 (MATLIS $[10$, p. 60]). Let $R$ be a (commutative) valuation ring with quotient field $Q$. Then $R$ is almost maximal if and only if $Q / R$ is an injective $R$ module. In this case, $Q / I$ is an indecomposable, injective $R$-module for every ideal $I$ of $R$.

COROLlary 3. Let $1 \leqq n \leqq \infty$. Then there exists a valuation ring $R$ such that $\sup \{\operatorname{id}(I) \mid I$ a right ideal of $R\}=\sup \{\operatorname{id}(R / I) \mid I$ a right ideal of $R\}=1$, and $\operatorname{gl} . \mathrm{d}(R)=n$.

Proof. Assume $R$ is almost maximal. Then, for any ideal $I$ of $R$,

$$
0 \rightarrow I \rightarrow Q \rightarrow Q / I \rightarrow 0 \quad(0 \rightarrow R / I \rightarrow Q / I \rightarrow Q / R \rightarrow 0)
$$

is an injective resolution of $I(R / I)$ by Lemma 10. Hence $\operatorname{id}(I)=1(=\operatorname{id}(R / I))$ if $I \neq 0(I \neq R)$. The rings constructed in Corollary 2 were maximal, hence almost 
maximal, for $n \geqq 2$. Hence the ring $R$ may have any global dimension, even though the supremum of the global dimensions of its cyclic modules and of its ideals $\leqq 1$.

In the proof of Theorem A, we need only the following conditions on $R$ and $I$ :

(i) Every projective $R$-module is free.

(ii) For all $i \in I, r \in R, i r=0 \Leftrightarrow i=0$ or $r=0$.

(iii) $I=\bigcup_{\beta<\alpha} x_{\beta} R$, where $x_{\beta} R \supset x_{y} R$ for all $\beta>\gamma$.

Thus Theorem $\mathrm{A}$ is true for any ring $R$ and module $I$ having these properties. In particular, if $R$ is a two-sided valuation ring, Theorem $A$ applies to any fractional ideal ( $R$-submodule) of the quotient field $Q$ of $R$. Then $\operatorname{pd}\left(Q_{R}\right) \leqq n+1 \Leftrightarrow Q_{R}$ is generated by some set of cardinality $\leqq \boldsymbol{\aleph}_{n}$. For the case $n=0$, this was noted by Kaplansky. (See Matlis [11, p. 386].) In [9], Kaplansky shows that any commutative domain with a unique maximal ideal has $\operatorname{pd}(Q) \leqq 1 \Leftrightarrow Q$ is countably generated. It would be interesting to know what modifications of Kaplansky's hypotheses imply $\operatorname{pd}(Q) \leqq n+1 \Leftrightarrow Q$ is $\aleph_{n}$-generated.

3. Global dimension of right perfect rings. By Corollary 3 to Theorem A, one cannot generalize the Global Dimension Theorem to a statement about injective dimensions of ideals or cyclics for an arbitrary ring $R$. However, if $R$ is right perfect, it is possible to prove an injective global dimension theorem.

Definition. We say that a module $M$ is $B$-projective for $B \in \mathfrak{M}_{R}$ if for every exact sequence $\mu: B \rightarrow C \rightarrow 0$, and every $f \in \operatorname{Hom}_{R}(M, C)$, there is a $g \in \operatorname{Hom}_{R}(M, B)$ such that $\mu g=f$.

Definition. A ring $R$ is right perfect if every right $R$-module has a projective cover. (See Bass [2].)

THEOREM (SANDOMIERSKI). Let $M^{\prime} \rightarrow M \rightarrow 0$ be a projective cover of $M \in \mathfrak{M}_{R}$. If $M$ is $B_{i}$-projective for all $B_{i}, i \in \mathscr{I}$, then $M$ is $\sum_{i \in \mathscr{S}} B_{i}$-projective.

Proof. This is a combination of Theorems 4.4 and 4.1 in Sandomierski [14].

COROLlaRY. If $R$ is right perfect, then $M \in \mathfrak{M}_{R}$ is $R$-projective if and only if $M$ is projective.

Proof. If $M$ is projective, then it is $B$-projective for all $B \in \mathfrak{M}_{R}$.

Now assume $M$ is $R$-projective. Since $M$ has a projective cover, by Sandomierski's theorem, $M$ is $F$-projective for any free module $F$. Since there exists a free module $F$ and an epimorphism $\mu: F \rightarrow M$, there is a map $\pi: M \rightarrow F$ such that $\mu \pi=1_{M}$, the identity map of $M$. Then $M$ is a direct summand of $F$ and so projective.

Thus, for right perfect rings $R, R_{R}$ is a test module for projectivity. We now parallel the steps used to prove the Global Dimension Theorem, using injective, rather than projective dimension, and dualizing other notions.

Lemma 11. The following conditions are equivalent for the right perfect ring $R$ :

(i) $P$ is a projective $R$-module.

(ii) $\operatorname{Ext}^{1}(P, I)=0$ for all right ideals $I$ of $R$. 
Proof. (i) $\Rightarrow$ (ii) is well known. (See for example, Jans [7, p. 45].)

(ii) $\Rightarrow$ (i). The exact sequence $0 \rightarrow I \rightarrow R \rightarrow R / I \rightarrow 0$ induces an exact sequence

$$
0 \rightarrow \operatorname{Hom}_{R}(P, I) \rightarrow \operatorname{Hom}_{R}(P, R) \rightarrow \operatorname{Hom}_{R}(P, R / I) \rightarrow \operatorname{Ext}^{1}(P, I) \rightarrow \cdots .
$$

If, for all $I, \operatorname{Ext}^{1}(P, I)=0$, this states that $P$ is $R$-projective. By the corollary to Sandomierski's theorem, $P$ is projective.

LEMMA 12. The following are equivalent for the right perfect ring $R$ and $M \in \mathfrak{M}_{R}$ :

(i) $\operatorname{pd}(M) \leqq n$.

(ii) $\operatorname{Ext}^{n+1}(M, I)=0$ for all right ideals $I$.

Proof. (i) $\Rightarrow$ (ii). $\operatorname{Ext}^{n+1}(M, \quad)=0$ is well known. (See Jans [7, p. 48].)

(ii) $\Rightarrow$ (i). If $\mathscr{P}$ is a projective resolution for $M$, then for any module $N$, $\operatorname{Ext}^{n+1}(M, N) \approx \operatorname{Ext}^{1}$ (image $\left.d_{n}, N\right)$. (See [7, p. 47].) Then, for all right ideals $I$, $\operatorname{Ext}^{n+1}(M, I)=0 \Leftrightarrow \operatorname{Ext}^{1}$ (image $\left.d_{n}, I\right)=0 \Leftrightarrow$ image $d_{n}$ is projective $\Leftrightarrow \operatorname{pd}(M) \leqq n$.

LEMMA 13. Let $R$ be a right perfect ring with Jacobson radical J. Then

$$
\operatorname{gl} \mathrm{d}(R)=\mathrm{id}(R / J) .
$$

Proof. This is an immediate consequence of Eilenberg [5, Theorem 12].

THEOREM B. Let $R$ be a right perfect ring. Then

$\sup \{\operatorname{id}(I) \mid I$ a right ideal of $R\} \stackrel{\circ}{=} \operatorname{gl} . \mathrm{d}(R)=\sup \{\operatorname{id}(R / I) \mid I$ a right ideal of $R\}$.

Proof. By Lemma 13, the second equality holds.

Let $\sup \{\operatorname{id}(I) \mid I$ a right ideal of $R\}=n$. If $n=\infty$, we are done. If not, for every $I$, $\operatorname{Ext}^{n+1}(, I)$ is the zero functor. By Lemma 12 , for every $M \in \mathfrak{M}_{R}, \operatorname{pd}(M) \leqq n$, so $\operatorname{gl} . \mathrm{d}(R) \leqq n$. Clearly $\operatorname{gl} . \mathrm{d}(R) \geqq n$, so $\operatorname{gl} . \mathrm{d}(R)=n$.

We note that, for $M \in \mathfrak{M}_{R}$, the exact sequence

$$
0 \rightarrow J \rightarrow R \rightarrow R / J \rightarrow 0
$$

induces an exact sequence

$$
\cdots \rightarrow \operatorname{Ext}^{m}(M, J) \rightarrow \operatorname{Ext}^{m}(M, R) \rightarrow \operatorname{Ext}^{m}(M, R / J) \rightarrow \operatorname{Ext}^{m+1}(M, J) \rightarrow \cdots .
$$

If $\operatorname{gl} . \mathrm{d}(R)=n<\infty$, by Lemma 13 there exists an $M$ such that $\operatorname{Ext}^{n}(M, R / J) \neq 0$. Since $\operatorname{Ext}^{n+1}(M, J)=0, \operatorname{Ext}^{n}(M, R) \neq 0$, so id $(R) \geqq n$. Then $\operatorname{id}(R)=n$.

Although $R_{R}$ need not be a test module for projectivity for Noetherian rings, we can prove a global dimension theorem for Noetherian rings comparable to Theorem B.

LEMMA 14. For any ring $R, \sup \{\operatorname{id}(R / I) \mid I$ a right ideal $\}=\sup \{\operatorname{id}(M) \mid M$ is a finitely generated right $R$-module\}.

Proof. We use induction on the number of generators of $M$. Let $\sup \{\operatorname{id}(R / I)\}=n$. If $n=\infty$, we are done. Hence assume $n<\infty$. If $M$ is generated by 1 element, then 
$M$ is isomorphic to $R / I$ for some right ideal $I$, so $\operatorname{id}(M) \leqq n$. Now assume that $M$ generated by $k$ elements implies $\operatorname{id}(M) \leqq n$. Let $M^{\prime}=\sum_{i=1}^{k+1} x_{i} R$. The sequence

$$
0 \rightarrow N=\sum_{i=1}^{k} x_{i} R \rightarrow M \rightarrow x_{k+1} R / x_{k+1} R \cap N=P \rightarrow 0
$$

is exact and induces an exact sequence

$$
\cdots \rightarrow \operatorname{Ext}^{n+1}(A, N) \rightarrow \operatorname{Ext}^{n+1}(A, M) \rightarrow \operatorname{Ext}^{n+1}(A, P) \rightarrow \cdots
$$

for all $A \in \mathfrak{M}_{R}$. $\operatorname{Ext}^{n+1}(, N)=0$ by the induction hypothesis, $\operatorname{Ext}^{n+1}(, P)=0$ since $P$ is cyclic. Hence $\operatorname{Ext}^{n+1}(, M)=0$, so $\operatorname{id}(M) \leqq n$. Thus $\sup \{\operatorname{id}(M) \mid M$ is finitely generated $\} \leqq n$.

The reverse inequality is obvious, so the lemma follows.

LEMMA 15. If $R$ is right Noetherian, then a direct limit of modules of injective dimension $\leqq n$ has injective dimension $\leqq n$.

Proof. See Bass [3, p. 19].

LEMMA 16. If $R$ is right Noetherian, and if $\mathrm{id}(R)$ and the finitistic injective dimension of $R$ are both finite, then they are equal.

Proof. See Bass [3, p. 24].

THEOREM C. If $R$ is right Noetherian, then $\sup \{\operatorname{id}(R / I) \mid I$ a right ideal of $R\}$ $=\operatorname{gl} . \mathrm{d}(R)=\sup \{\operatorname{id}(I) \mid I$ a right ideal of $R\}$.

Proof. Let $\sup \{\operatorname{id}(R / I)\}=n$. By Lemma $14, n=\sup \{\operatorname{id}(M) \mid M$ is finitely generated\}. Since any module is the direct limit of its finitely generated submodules, by Lemma $15, \operatorname{id}(M) \leqq n$ for any module $M$. Hence gl.d $(R) \leqq n$. Clearly gl.d $(R) \geqq n$, so $\operatorname{gl} . \mathrm{d}(R)=n$.

If $\operatorname{gl} . \mathrm{d}(R)<\infty$, by Lemma $16, \operatorname{gl} . \mathrm{d}(R)=\mathrm{id}(R) \leqq \sup \{\operatorname{id}(I) \mid I$ a right ideal of $R\}$ since $R$ is a right ideal of itself. Since the reverse inequality is clear, $\operatorname{gl} . \mathrm{d}(R)$ $=\sup \{\operatorname{id}(I) \mid I$ a right ideal of $R\}$.

If $\operatorname{gl} . \mathrm{d}(R)=\infty$, and $\sup \{\operatorname{id}(I) \mid I$ a right ideal of $R\}=m$ is finite, the exact sequence $0 \rightarrow I \rightarrow R \rightarrow R / I \rightarrow 0$ involves two modules, $I$ and $R$, of injective dimension $\leqq m$. Hence id $(R / I) \leqq m+1$ by the induced exact sequence of Ext. But, by the first part of the theorem, this implies gl.d $(R) \leqq m+1$, a contradiction. Thus

$$
\operatorname{gl} . \mathrm{d}(R)=\sup \{\operatorname{id}(I) \mid I \text { a right ideal of } R\}=\infty .
$$

\section{REFERENCES}

1. M. Auslander, On the dimension of modules and algebras. III, Nagoya Math. J. 9 (1955), 67-77.

2. H. Bass, Finitistic dimension and a homological generalization of semi-primary rings, Trans. Amer. Math. Soc. 95 (1960), 466-488.

3. - Injective dimension in noetherian rings, Trans. Amer. Math. Soc. 102 (1962), 18-29. 
4. H. Cartan and S. Eilenberg, Homological algebra, Princeton Univ. Press, Princeton, N. J., 1956.

5. S. Eilenberg, Homological dimension and syzygies, Ann. of Math. 64 (1956), 328-336.

6. P. Halmos, Naive set theory, Van Nostrand, Princeton, N. J., 1960.

7. J. P. Jans, Rings and homology, Holt, Rinehart and Winston, New York, 1964.

8. I. Kaplansky, Projective modules, Ann. of Math. 68 (1958), 372-377.

9. - The homological dimension of a quotient field, Nagoya Math. J. 27 (1966), 139-142.

10. E. Matlis, Injective modules over Prüfer rings, Nagoya Math. J. 15 (1959), 57-69.

11. - Divisible modules, Proc. Amer. Math. Soc. 11 (1960), 385-391.

12. O. Ore, Linear equations in non-commutative fields, Ann. of Math. (2) 32 (1931), 463, 477.

13. B. L. Osofsky, Rings all of whose finitely generated modules are injective, Pacific J. Math. 14 (1964), 645-650.

14. F. Sandomierski, Relative injectivity and projectivity, Ph.D. Thesis, Penn. State University, University Park, 1964.

15. O. F. G. Schilling, The theory of valuations, Math. Surveys No. 4, Amer. Math. Soc., Providence, R. I., 1950.

16. W. Sierpinski, Cardinal and ordinal numbers, Hafner, New York, 1958.

17. A. Tarski, Uber unerreichbare Kardinalzahlen, Fund. Math. 30 (1938), 68-89.

\section{Rutgers, The State University,}

New Brunswick, New Jersey 